\title{
Outcome Following Ultrasound Guided Foam Sclerotherapy Treatment for Varicose Veins in a Tertiary Care Centre
}

\author{
Mayurika Singh ${ }^{1}$, Anish Kola ${ }^{2}$, lla Katyayan ${ }^{3}$, Soumitra Manwatkar ${ }^{4}$ \\ ${ }^{1}$ Assistant Professor, PG Department of Radio-Diagnosis, M.L.N Medical College, Allahabad, ${ }^{2} J u n i o r$ Resident, PG Department \\ of General Surgery, M.L.N Medical College, Allahabad, ${ }^{3}$ Junior Resident, PG Department of General Surgery, M.L.N Medical

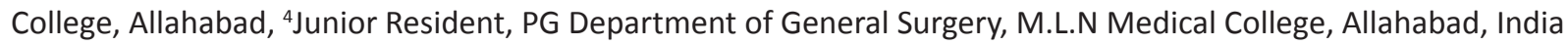

Corresponding author: Dr. Anish Kola, Junior Resident, PG Department of General Surgery, M.L.N Medical College, Allahabad, India

DOI: http://dx.doi.org/10.21276/ijcmsr.2018.3.4.19

How to cite this article: Mayurika Singh, Anish Kola, Ila Katyayan, Soumitra Manwatkar. Outcome following ultrasound guided foam sclerotherapy treatment for varicose veins in a tertiary care centre. International Journal of Contemporary Medicine Surgery and Radiology. 2018;3(4):D82-D86.

\section{A B S T R A C T}

Introduction: Patients with varicose veins may present with dilated, elongated and tortuous superficial veins of the limbs with complaints of dull aching pain, heaviness, discomfort, and extremity fatigue. Patients with symptoms have difficulty in daily activities such as work, recreation. Such patients are subjected to sclerotherapy injection by ultrasound guided which is minimally invasive technique that allows patients to rapidly return to their baseline activity level. Objective: To evaluate the outcomes of the foam sclerotherapy (UGFS) treatment under ultrasound guidance for varicose vein.

Material and Methods: patients with varicose veins were selected and treated with sodium tetradecyl sulphate as sclerosant and followed up to 1-year. Total 185 legs in 148 patients, 111 unilateral limbs, and 74 bilateral limbs were treated by this method.

Results: Out of 185 legs saphenofemoral junction (SFJ) incompetence was found in 57 and sapheno-popliteal junction (SPJ) incompetence in 41, perforator's incompetence in 21, while combination of SFJ and perforators and SPJ and perforators in 19 and 14, respectively. Great saphenous vein varicosity was found in 87, short saphenous vein in 54 and others 44 . Early outcome was $100 \%$ success rate. No recurrences were noted at 1-year follow-up. Early complications were: Superficial skin necrosis in 7 legs (3.78\%), pain at injection sites in 27 legs (14.59\%), superficial thrombophlebitis in injected vein in 23 legs (12.43\%), and skin staining around injected veins in 16 legs (8.64\%).

Conclusion: UGFS is a safe, comparatively easy, and beneficial for the patients with varicose veins and found to be associated with less complications like skin staining and pain.

Key words: Foam Sclerotherapy, Ultrasound Guided Foam Sclerotherapy Outcome, Ultrasound Guided Sclerotherapy, Varicose Veins

\section{INTRODUCTION}

UGS was described in 1989 as a treatment for the superficial axial system. Since then, the use of UGS has expanded to treatment of incompetent perforator branches and large venous tributaries. Sclerosants act to disrupt the venous endothelium, causing a periphlebitic reaction, which acts to obliterate the vein segment. Sclerosants mainly causes endothelial damage which reveals sub-endothelial collagen fibres and the intrinsic coagulation pathways are activated and cause inflammatory reactions that occur at the vessel wall and thrombus formation and maturation in the vessel lumen which results in fibrosis, and obliteration of the vessels. ${ }^{1-5}$ various sclerosing agents include $3 \%$ sodium tetra decyl sulphate, ethoxy scleral, 5\%ethanolamine oleate. The blood deactivates the action of the sclerosing agent, dose to adjusted to avoid adverse effects, which led to the development of UGFS (Ultrasound Guided Foam Sclerotherapy)

Foams have several benefits over liquid sclerosants, like a large dose of foam can be used in a single session, have large surface area leading to greatest efficacy, displaces blood and prevents dilution, and inactivation of the sclerosants. Foams are visible on duplex ultrasound, and it is possible to manipulate the foam once it has been injected into the vessels.

The aim of the study was to compare the outcome of UGFS (minimally-invasive) over the surgical procedure with regards to complications following treatment in patients with varicose vein of the lower limb.

\section{MATERIAL AND METHODS}

The study was carried out in Swaroop Rani Nehru Hospital attached to M.L.N.Medical College Allahabad, after taking approval from the ethical committee and obtaining written and informed consents from the patients, between June 2017 and July 2018. The patients details were recorded for the new modality of treatment procedure. Every patient referred with 
varicosity of the lower limb (varicose vein) were explained different modalities of treatments available at the hospital. The treatment modalities available were sclerotherapy and invasive surgical methods in the hospital. Other modern minimally invasive methods were not available in our hospital. After a full discussion of the available options to the patients and based on their requirements, ultrasound guided foam sclerotherapy (UGFS) seems to be selected by most of the patients, particularly those with strong cosmetic concern and army personnel. Patients included in the study were those having great saphenous vein (GSV), small saphenous vein (SSV), other recurrent veins with significant incompetent deep venous communication [Fig-1].

\section{Clinical assessment}

The full history and clinical examination of the patient was done who were undergoing UGFS treatment including palpation of peripheral pulses. Patients with, having deep vein thrombosis (DVT), deep venous insufficiency and $A-V$ malformations, were excluded.

\section{Initial duplex ultrasound assessment}

All patients with varicosity were initially subjected to duplex ultrasonography to examine superficial venous system and deep venous system in both standing and sitting position on the table. In standing position with their weight on the contra-lateral limb and the leg to be examined was kept slightly bent with the heel on the floor to relax the calf muscles. The veins and the venous segments were assessed were: the entire venous system of lower limb from the SF junction and sapheno-popliteal junction (SPJ), the whole length of the GSV, SSV, and competency of perforators were assessed. The diameter and reflux were assessed and were considered pathological when it exceeded $0.5 \mathrm{~s}$.

\section{Procedure}

\section{Cannulation and injection}

Before initiating the treatment, in the standing posture veins were marked then cannulated under ultrasound guidance, depending on the size, depth, and tortuosity of the veins. For GSV, cannulation was done just below or above the level of knee joint. For Other veins distal most accessible sites were used. The skin sensitivity of sclerosant was done before the use of drug.

Foam was made by connecting two $5 \mathrm{ml}$ syringe to a triway and using $1 \mathrm{ml}$ of sclerosant with $4 \mathrm{ml}$ of air [Fig.2], leg was elevated about $45^{\circ}$ and foam was introduced under ultrasound guidance in to the vein, where lower limb was elevated 40-50 degree angle. Maximum of $2 \mathrm{ml}$ foam was injected per cannula. To increase the venous flow of the lower limb the patient was asked to plantar and dorsiflex the ankle. A maximum15- $20 \mathrm{ml}$ foam was used in a single session.

\section{Bandaging and compression}

After completing the foam injection, all cannulas were removed and crepe (elastic) bandage was applied to the limb [Fig. 4]. Too much pressure was avoided to prevent any vascular compromise to leg. after 3-5 days the compression bandage was removed and looked for any residual varicosities and complications. Patient was advised stocking or compression bandage while walking running and was advised limb elevation in night.

\section{Follow-up}

All patients were followed-up on a regular basis until one year. During follow up the complete examination was done and reviewed in the symptoms and varicosities any complications and any signs of DVT. Benefits of the treatment was assessed on the basis of improvement in signs and symptoms.

\section{DATA ANALYSIS AND RESULTS}

Total 185 legs in 148 patients (101 males and 47 females), 111 unilateral limbs, and 37 bilateral limbs were treated by this method for different types of lower limb varicosities [Table 1]. Out of 148 legs SFJ incompetence was found in 57 and SPJ incompetence in 41, perforator's incompetence in 27, while combination of SFJ and perforators and SPJ and perforators in 19 and 14 respectively [Table 2]. GSV varicosity was found in 87 , short saphenous vein in 54 and others 44.43 limbs had recurrent varicose veins previously treated by other modalities, and 142 limbs had primary

\begin{tabular}{|l|c|c|c|c|c|c|}
\hline Variables & Total no. patients & Male & Female & Total limbs & Bilateral limbs & Unilateral limbs \\
\hline Number & 148 & 106 & 42 & 185 & 37 & 111 \\
\hline \multicolumn{7}{|c|}{ Table-1: Patient demographics } \\
\hline
\end{tabular}

\begin{tabular}{|l|c|c|c|c|c|c|}
\hline Incompetence & SFJ & SPJ & Perforator's & SFJ and perforator's & SPJ and perforator's & No \\
\hline Number & 57 & 41 & 21 & 19 & 14 & 33 \\
\hline \multicolumn{7}{|c|}{ Table-2: Duplex findings } \\
\hline
\end{tabular}

\begin{tabular}{|l|c|c|c|c|c|c|c|}
\hline Varicosities & GSV & SSV & Others & Primary & Recurrent & Uncomplicated & Complicated \\
\hline Numbers & 87 & 54 & 44 & 142 & 43 & 120 & 65 \\
\hline GSV: Great saphenous vein; SSV: Small saphenous vein \\
\hline \multicolumn{7}{|c|}{ Table-3: Location of varicosities } \\
\hline
\end{tabular}

\begin{tabular}{|l|c|c|c|c|c|c|c|}
\hline Complications & $\begin{array}{c}\text { Superficial } \\
\text { skin necrosis }\end{array}$ & $\begin{array}{c}\text { Pain at } \\
\text { injection sites }\end{array}$ & Bruising & DVT & SVT & $\begin{array}{c}\text { Superficial } \\
\text { thrombhophlebitis }\end{array}$ & Skin staining \\
\hline Numbers(\%) & $7(3.78 \%)$ & $27(14.59 \%)$ & $14(7.56 \%)$ & 0 & $9(4.86 \%)$ & $23(12.43 \%)$ & $16(8.64 \%)$ \\
\hline \multicolumn{7}{|c|}{ Table-4: Complications } \\
\hline
\end{tabular}




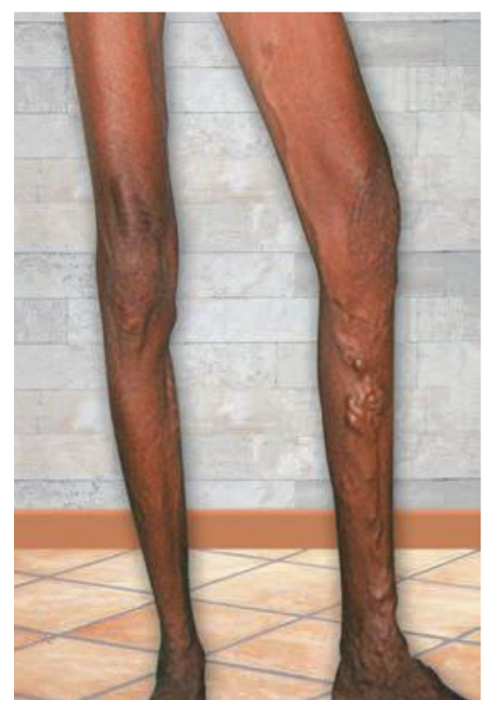

Figure-1: Varicosity of great saphenous vein

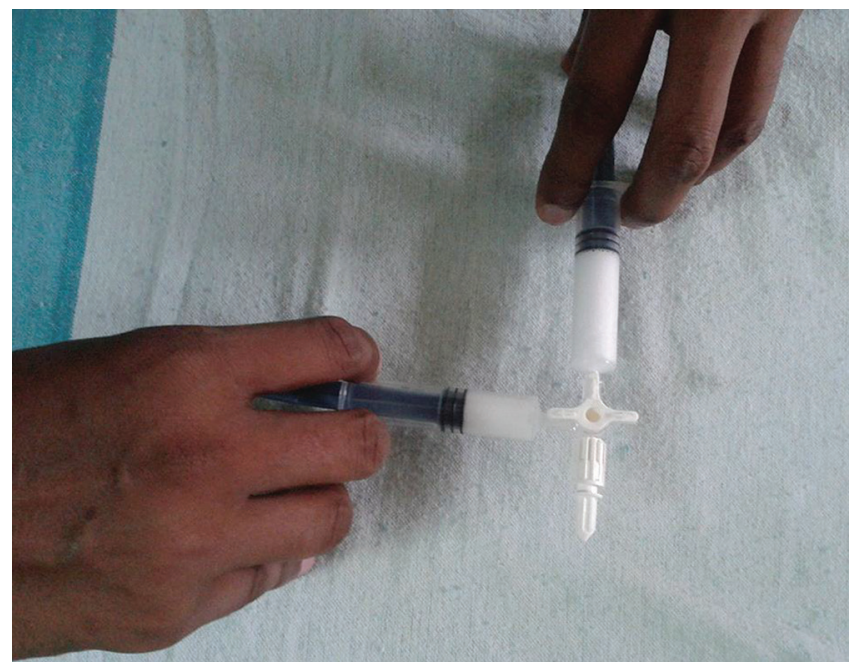

Figure-2: Technique of foam preparation using two syringes and one triway
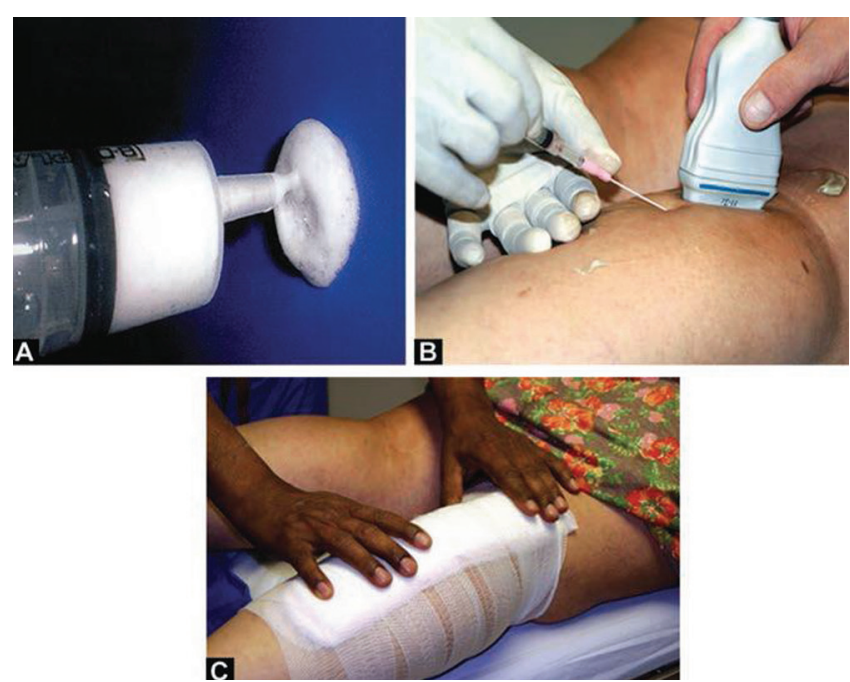

Figure-3: Procedures in foam sclerotherapy

varicose veins. 65 were treated for complicated varicose veins and 120 limbs for uncomplicated varicose veins Table 3. On first follow-up visit after 3-5 days, all varicosities treated with this method had disappeared. Early outcome was $100 \%$.

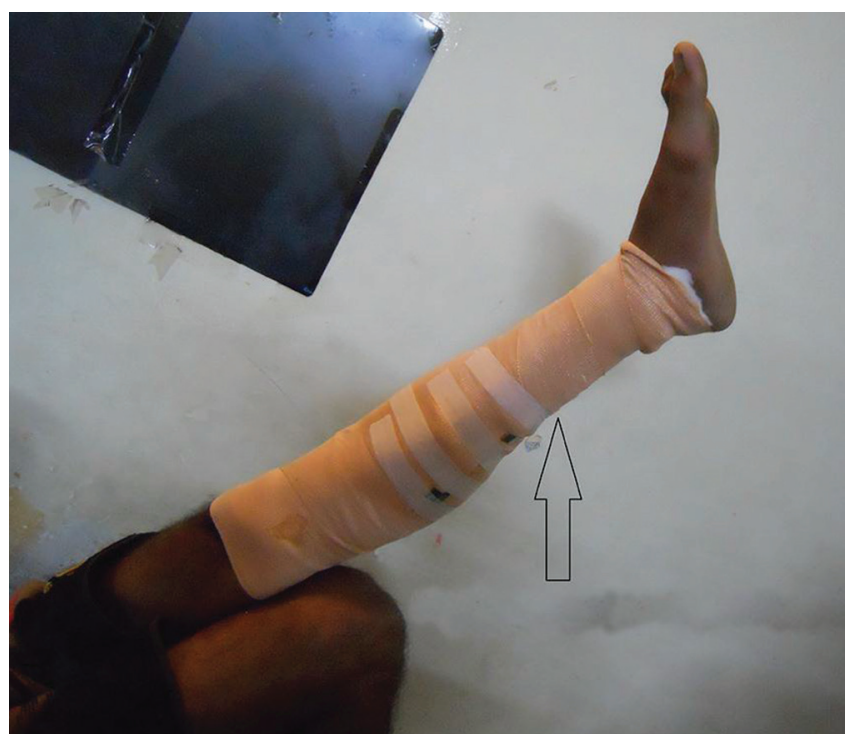

Figure-4: Bandaging

Early complications were: Superficial skin necrosis in 7 legs at one or two injection sites (3.78\%). Pain at injection sites in 27 legs (14.59\%), superficial thrombophlebitis in injected vein in 23 of the 185 legs (12.43\%), bruising was noted in 14 legs $(7.56 \%)$, and skin staining around injected veins were found in 16 legs (8.64\%). Only 9 of the 185 legs developed superficial vein thrombosis (4.86\%), which was treated with NSAIDS, rest, limb elevation and stockings Table 4. For all these complications, no treatment was required, and they disappeared without any specific treatment. At the end of 6 months and 1-year, no complications and recurrences were found.

\section{DISCUSSION}

When results of the study was compared with other conventional methods such as saphenous vein stripping, UGFS is found to be associated with lesser pain, very much-tolerable and safe, and no DVT, or any allergic reaction, infection, headache, and blurring of vision. Bruising was noted in 14 legs (7.56\%) in this study which was less than other methods like saphenous vein stripping (SVS). The incidence of bruising with SVS was found to be $25-30 \%$ reported in different other studies. ${ }^{6,7}$ With UGFS treatment bruising and pigmentation was found to be $26-30 \%$ in various studies $^{.8,9-12}$ After RFA, it is about $13-27 \% \%^{7,13}$ and after EVLA, it is $11-15 \%$. $^{14,15}$

According to Kalodiki et al. ${ }^{16}$ and Shadid et al. ${ }^{17}$ surgery is associated with the lesser incidence of pigmentation (5\% and $1.1 \%$ respectively) in comparison to UGFS (15\% and 5.6\%, respectively). Improvements in symptoms and quality of life were $100 \%$ in our study which was found to be similar to other studies. ${ }^{18}$ In terms of expectations, one study shows that exceeded in $25 \%$ while unmet in $10 \% .^{19}$

Recurrences associated with UGFS treatment varies from $4.9 \%$ to $40 \% \%^{8,20-23}$ but there were no recurrences seen in our study. Treatment failures associated with UGFS treatment was $2 \%{ }^{8}$ and $57 \%,{ }^{12}$ but there were no failure in our study conducted in SRN Hospital. Anaphylaxis is an established complication of liquid sclerotherapy, but can occur with foam also. No cases of anaphylaxis was recorded in our study like 
other studies. ${ }^{9}$ Bradbury et al. ${ }^{3}$ reported allergy to the foam in $0.1 \%$ of patients in their study.

DVT and thromboembolism after UGFS is a very rare complication and reported in $<1 \%$ of the patients. ${ }^{3,8,9,27,28} \mathrm{No}$ incidence of DVT was noted in this study and also according to Brunken et al. ${ }^{10}$ and Hamahata et al., ${ }^{24}$ Figueiredo et al. ${ }^{12}$ reported the occurrence of DVT in $9 \%$ of their patients. Later on it was found that using foam volume more than 10 $\mathrm{ml}$ in single limb resulted in 3-fold chances of $\mathrm{DVT}^{25}$ and increased production of endothelin-1 is associated with high chances of DVT. ${ }^{31}$ Scurr et al. ${ }^{26}$ Brzoza et al., ${ }^{30}$ and Guex et al. ${ }^{29}$ each of them reported single case of allergic reactions in their studies.

After this study, Breu et al. ${ }^{32}$ and Hamel-Desnos et al. ${ }^{33}$ uses maximum $10 \mathrm{ml}$ of foam without any incident of DVT. Superficial thrombophlebitis incidence reported in different studies was found to be $<15 \% .{ }^{8,10}$

Different maneuvers have been described to improve the efficacy and safety of foam sclerotherapy by decreasing the amount of foam entering into the deep venous system and systemic circulation, like leg elevation before foam injection, blocking of SFJ and SPJ before injection. ${ }^{34}$ One study shows $39 \%$ incidence. ${ }^{35}$ In our study, it's incidence was about $12.43 \%$. Other systemic complications associated with UGFS were photopsia, transient blurring of vision, transient ischemic attack, headache, chest tightness, and dry cough has been reported in $<1 \%$ of the patients. ${ }^{3,8,9}$ No such complications noted in our study.

The drawback of the study was that there is no comparison and randomization between two groups. Only surgeons in our unit of general surgery of swaroop rani nehru hospital used UGFS as preferred treatment while others unit surgeons used conventional methods of treatment such as SVS, SFJ ligation, SPJ ligation, and perforator ligation. All patients in this study were referred from surgeons of our hospital through a common referral pool. Therefore, there was some clinical or surgeon selection bias.

Another limitation of our study was the size of UGFS cohorts, although this was unlikely to have resulted in any systematic bias. Assessment of complications and outcomes were objective and decided by the therapist. The observer bias could have been improved by subjective assessment of complications and outcome in the follow up, by using a validated questionnaire method and scoring system.

\section{CONCLUSION}

In comparison to the conventional invasive method UGFS seems to be associated with less complications and decrease recurrences with good cosmetic out come and cost effective and also safe to the patient. UGFS can be used in all varicosity of the lower limb including the perforators.

\section{REFERENCES}

1. Coleridge Smith P. Sclerotherapy and foam sclerotherapy for varicose veins. Phlebology 2009;24(1):260-9.

2. Darvall KA, Bate GR, Adam DJ, Bradbury AW. Recovery after ultrasound-guided foam sclerotherapy compared with conventional surgery for varicose veins. Br J Surg 2009;96 (3):1262-7.
3. Guex JJ, Schliephake DE, Otto J, Mako S, Allaert FA. The French polidocanol study on long-term side effects: A survey covering 3,357 patient years. Dermatol Surg 2010;36 Suppl 2:993-1003.

4. Kakkos SK, Bountouroglou DG, Azzam M, Kalodiki E, Daskalopoulos M, Geroulakos G. Effectiveness and safety of ultrasound-guided foam sclerotherapy for recurrent varicose veins: Immediate results. J Endovasc Ther 2006;13 (5):357-64.

5. Bradbury AW, Bate G, Pang K, Darvall KA, Adam DJ. Ultrasound-guided foam sclerotherapy is a safe and clinically effective treatment for superficial venous reflux. J Vasc Surg 2010;52 (2):939-45.

6. Lurie F, Creton D, Eklof B, Kabnick LS, Kistner RL, Pichot $\mathrm{O}$, et al. Prospective randomized study of endovenous radiofrequency obliteration (closure procedure) versus ligation and stripping in a selected patient population (EVOLVeS Study). J Vasc Surg 2003;38 (4):207-14.

7. Subramonia S, Lees T. Sensory abnormalities and bruising after long saphenous vein stripping: Impact on short-term quality of life.J Vasc Surg 2005;42 (5):510-4.

8. Chapman-Smith P, Browne A. Prospective five-year study of ultrasound-guided foam sclerotherapy in the treatment of great saphenous vein reflux. Phlebology 2009;24 (6):183-8.

9. Jia X, Mowatt G, Burr JM, Cassar K, Cook J, Fraser C. Systematic review of foam sclerotherapy for varicose veins. Br J Surg 2007;94 (3):925-36.

10. Brunken A, Rabe E, Pannier F. Changes in venous function after foam sclerotherapy of varicose veins. Phlebology 2009;24 (1):145-50.

11. Blaise S, Bosson JL, Diamand JM. Ultrasound-guided sclerotherapy of the great saphenous vein with $1 \%$ vs $3 \%$ polidocanol foam: A multicentre double-blind randomised trial with 3-year follow-up. Eur J Vasc Endovasc Surg 2010;39 (5):779-86.

12. Figueiredo M, de Araujo SP, Figueiredo MF. Late follow-up of saphenofemoral junction ligation combined with ultrasound-guided foam sclerotherapy in patients with venous ulcers. Ann Vasc Surg 2012;26 (1):977-81.

13. Vasquez MA, Wang J, Mahathanaruk M, Buczkowski G, Sprehe E, Dosluoglu HH. The utility of the Venous Clinical Severity Score in 682 limbs treated by radiofrequency saphenous vein ablation. J Vasc Surg 2007;45 (4):1008-14.

14. Rasmussen LH, Bjoern L, Lawaetz M, Blemings A, Lawaetz B, Eklof B. Randomized trial comparing endovenous laser ablation of the great saphenous vein with high ligation and stripping in patients with varicose veins: Short-term results. J Vasc Surg 2007;46 (3):308-15.

15. Christenson JT, Gueddi S, Gemayel G, Bounameaux H. Prospective randomized trial comparing endovenous laser ablation and surgery for treatment of primary great saphenous varicose veins with a 2-year follow-up.J Vasc Surg 2010;52 (1):1234-41.

16. Kalodiki E, Lattimer CR, Azzam M, Shawish E, Bountouroglou D, Geroulakos G. Long-term results of a randomized controlled trial on ultrasound-guided 
foam sclerotherapy combined with saphenofemoral ligation vs standard surgery for varicose veins. J Vasc Surg 2012;55 (1):451-7.

17. Shadid N, Ceulen R, Nelemans P, Dirksen C, Veraart J, Schurink GW, et al. Randomized clinical trial of ultrasound-guided foam sclerotherapy versus surgery for the incompetent great saphenous vein. Br J Surg 2012;99 (2):1062-70.

18. Tan VK, Abidin SZ, Tan SG. Medium-term results of ultrasonography-guided, catheter-assisted foam sclerotherapy of the long saphenous vein for treatment of varicose veins. Singapore Med J 2012;53 (3):91-4.

19. Darvall KA, Bate GR, Sam RC, Adam DJ, Silverman SH, Bradbury AW. Patients' expectations before and satisfaction after ultrasound guided foam sclerotherapy for varicose veins. Eur J Vasc Endovasc Surg 2009;38 (6):642-7.

20. Hahn M, Schulz T, Junger M. Outcome four years after transcatheter foam sclerotherapy of the greater saphenous vein. Phlebologie 2008;37 (5):237-40.

21. Leopardi D, Hoggan BL, Fitridge RA, Woodruff PW, Maddern GJ. Systematic review of treatments for varicose veins. Ann Vasc Surg 2009;23 (4):264-76.

22. Pang KH, Bate GR, Darvall KA, Adam DJ, Bradbury AW. Healing and recurrence rates following ultrasound-guided foam sclerotherapy of superficial venous reflux in patients with chronic venous ulceration. Eur J Vasc Endovasc Surg 2010;40 (1):790-5.

23. Rasmussen LH, Lawaetz M, Bjoern L, Vennits B, Blemings A, Eklof B. Randomized clinical trial comparing endovenous laser ablation, radiofrequency ablation, foam sclerotherapy and surgical stripping for great saphenous varicose veins. Br J Surg 2011;98 (2):1079-87.

24. Hamahata A, Yamaki T, Sakurai H. Outcomes of ultrasound-guided foam sclerotherapy for varicose veins of the lower extremities: A single center experience. Dermatol Surg 2011;37 (1):804-9.

25. Myers KA, Jolley D. Factors affecting the risk of deep venous occlusion after ultrasound-guided sclerotherapy for varicose veins. Eur J Vasc Endovasc Surg 2008;36 (4):602-5.

26. Scurr JR, Fisher RK, Wallace SB, Gilling-Smith GL. Anaphylaxis following foam sclerotherapy: A life threatening complication of non-invasive treatment for varicose veins. Eur J Vasc Endovasc Surg Extra 2007;13 (1):87-9.

27. Myers KA, Jolley D, Clough A, Kirwan J. Outcome of ultrasound-guided sclerotherapy for varicose veins: Medium-term results assessed by ultrasound surveillance. Eur J Vasc Endovasc Surg 2007;33 (8):116-21.

28. Gillet JL, Guedes JM, Guex JJ, Hamel-Desnos C, Schadeck M, Lauseker M, et al. Side-effects and complications of foam sclerotherapy of the great and small saphenous veins: A controlled multicentre prospective study including 1,025 patients. Phlebology 2009;24 (1):131-8.

29. Guex JJ, Schliephake DE, Otto J, Mako S, Allaert FA. The French polidocanol study on long-term side effects: A survey covering 3,357 patient years. Dermatol Surg
2010;36 Suppl 2:993-1003.

30. Brzoza Z, Kasperska-Zajac A, Rogala E, Rogala B. Anaphylactoid reaction after the use of sodium tetradecyl sulfate: A case report. Angiology 2007;58 (1):644-6.

31. Frullini A, Felice F, Burchielli S, Di Stefano R. High production of endothelin after foam sclerotherapy: A new pathogenetic hypothesis for neurological and visual disturbances after sclerotherapy. Phlebology 2011;26 (4):203-8.

32. Breu FX, Guggenbichler S, Wollmann JC. 2nd European consensus meeting on foam sclerotherapy 2006, Tegernsee, Germany. Vasa 2008;37 Suppl 71:1-29.

33. Hamel-Desnos C, Allaert FA. Liquid versus foam sclerotherapy. Phlebology 2009;24 (3):240-6.

34. O'Hare JL, Earnshaw JJ. The use of foam sclerotherapy for varicose veins: A survey of the members of the Vascular Society of Great Britain and Ireland. Eur J Vasc Endovasc Surg 2007;34 (3):232-5.

35. Figueiredo M, Araujo S, Barros N Jr, Miranda F Jr. Results of surgical treatment compared with ultrasound-guided foam sclerotherapy in patients with varicose veins: A prospective randomised study. Eur J Vasc Endovasc Surg 2009;38 (3):758-63

Source of Support: Nil; Conflict of Interest: None

Submitted: 20-09-2018; Accepted: 29-10-2018; Published online: 02-12-2018 